\title{
Cerebral Lateralization of the EEG During Perceptual-Motor Integration in Young Adults with Down Syndrome: A Descriptive Study
}

\author{
CHIH-CHIA (JJ) CHEN ${ }^{1}$ | SHANNON D. R. RINGENBACH${ }^{2}$ | ARIELLE BIWER ${ }^{2} \mid$ ABBIE RIEKENA ${ }^{2}$ \\ ${ }^{1}$ Department of Kinesiology, Mississippi State University $\left.\right|^{2}$ School of Nutrition and Health Promotion, Program of Exercise Science and Health Promotion, Arizona State \\ University
}

Correspondence to: Chen, C-C (JJ), Department of Kinesiology, Mississippi State University, 216 McCarthy Gymnasium, MS, USA 39762,

Email: chih.chia.chen@msstate.edu

\author{
AT A GLANCE \\ Atypical cerebral specialization accounts for \\ the perceptual-motor integration deficits in \\ persons with Down syndrome (DS). The \\ purpose of current study was to test EEG alpha \\ power spectrum output in their brain's primary \\ auditory areas while performing unimanual \\ drumming under verbal, rhythm and melody \\ instructions. The results indicated that persons \\ with DS exhibited atypical specialization in \\ verbal instruction and the similar cerebral \\ specialization in rhythm and melody in \\ instructions compared to typical populations.
}

\begin{abstract}
BACKGROUND AND AIM: This study was aimed at investigating cerebral laterality of perceptual-motor integration in persons with Down syndrome (DS).

METHOD: Fourteen persons with DS between the ages of 12-39 drummed with their dominant hand (e.g., right hand) following verbal (i.e., drumming to a voice saying "drum"), rhythm (i.e., drumming to the sound of a drum being hit) and melody (i.e., drumming to the loudest beat) instructions. Electroencephalogram (EEG) data at T3 (left hemisphere) and T4 (right hemisphere) was collected and computed as cerebral specialization coefficients during drumming performance.

RESULTS: Our results were consistent with the model of atypical hemisphere processing of verbal information in the right hemisphere in persons with DS, which is opposite to the typical population (Elliott et al., 1987). In addition, the results showed that melody instructions were right hemisphere specialized and rhythm instruction was left hemisphere specialized in persons with DS.

CONCLUSION: This is the first study to systematically examine verbal, rhythm and melody processing in persons with DS. Rhythm and melody are two main components of music. Therefore, these results are promising for understanding mechanisms underlying cerebral processing as well as music therapy for persons with DS.
\end{abstract}

\section{INTRODUCTION}

Down syndrome (DS) is one of the most prevalent conditions associated with intellectual disabilities (ID). ${ }^{1}$ Over the last 20 years, there has been an increase in neurobehavioral research investigating perceptual-motor behavior for persons with DS. Many noninvasive neuropsychological protocols used behavioral measures and suggested that persons with DS exhibited left ear/right hemisphere lateralization for verbal information, which was opposite to the typical population (i.e., right ear/left hemisphere lateralization), and the organization and control of movement, including speech production, in left hemisphere. ${ }^{2-5}$ Thus, it is believed that this cost of inter-hemispheric communication further leads to verbal-motor difficulties in persons with DS. ${ }^{6}$

Furthermore, it is consistent with the results of behavioral research that Weeks, Chua, Weinberg, Elliott, and Cheyne (2002) conducted a single subject study with magnetoencephalography (MEG) and found that the participants with DS appeared to be more right hemisphere, rather than left hemisphere, specialized for language processing. ${ }^{7}$ However, using EEG technology, Groen, Alku, and Bishop (2008) analyzed temporal event-related potentials (ERPs) to understand lateralized processing of verbal stimuli in persons with DS ${ }^{8}$ and found that persons with DS were similarly sensitive to verbal stimuli on both temporal locations. Therefore, there is still a need to understand this model of 
atypical cerebral lateralization in persons with DS in order to improve their perceptualmotor performance.

Moreover, it has been reported that people with DS have an affinity to musicbased activity. ${ }^{9}$ In addition, Ringenbach, Gibbons, Chen, and Mulvey (2010) investigated the influence of music (i.e., melody) on motor performance which revealed that adults with DS performed faster and more consistently with music than verbal and rhythm instructions. ${ }^{10}$ However, despite the observed love of music and potential therapeutic benefits of music on motor performance in people with DS, there is remarkably little research using music with this population.

In summary, the innovation of this study is that it will be one of the few studies to examine cerebral laterality of perceptual-motor integration in persons with DS. Electroencephalogram (EEG) power spectral analysis is a well-established method for the analysis of cerebral lateralization. ${ }^{11}$ We used this direct measure of brain activation to examine whether EEG data was consistent with the atypical model of verbal lateralization in persons with DS. In addition, it has been reported that music is processed in both cerebral hemispheres (e.g., rhythm in the left and melody in the right hemisphere) in typical populations. ${ }^{12,13}$ Hence, we predicted that there is a possibility that persons with DS may be atypical in musical processing as well.

\section{METHODS}

\section{Participants}

There were fourteen participants with DS, aged 12 to 39 years (mean chronological age was 21.57 years and mean mental age was 5.49 years) in this study. Participants were recruited from a variety of local DS organizations (e.g., Sharing Down syndrome Arizona, DS Network Arizona, and Special Olympic Arizona). All protocols were approved by the Human Subjects Institutional Review Board of Arizona State University.

\section{Procedures}

Upon arriving, participants read (or were read) and signed the informed consent or assent forms and the parent or guardian signed a consent form. Next, participants were tested for handedness using a seven-item handedness inventory. ${ }^{14}$ The mental ages of participants were collected using the Peabody Picture Vocabulary Test (3rd ed.; PPVT-III). Hearing was assessed with an audiometer (Maico Ma 25). Vision was tested using the Kindergarten Snellen eye chart (i.e., symbols; sailboat, star, moon) for all participants, regardless of whether they could recognize letters or not. All participants had acceptable vision, hearing, and no known neurological disorders.

\section{Task and Apparatus}

Continuous unimanual drumming movements were performed with the dominant right hand on a drum using an overhand grip. The presentation order of the three types of stimuli was counterbalanced across participants. 


\section{Verbal}

The computer said "drum" every $500 \mathrm{~ms}$, and participants were instructed to drum until the researcher asked them to stop.

\section{Rhythm}

The computer played the sound of a drum being hit every 500 ms. Participants were instructed to hit the drum at the same time as the sound, until they were asked to stop.

\section{Melody}

Pentatonic folk music style in 2/4 meter was played continuously. The participants were instructed to hit the drum to the loudest beat in the music. Twelve pretest trials were conducted on all participants for each instruction type (e.g., verbal, rhythm and melody). The participants were instructed about what they would hear and how to drum.

There were three trials for each instruction type, and each trial lasted as long as it took to get twelve seconds of data on the EEG computer without the disruption of blinking.

\section{EEG Data Collection and Reduction}

For all participants, EEG electrodes were attached to the participant's head. EEG was collected from T3 and T4 using the International 10-20 system. The sampling rate for all data was $1000 \mathrm{~Hz}$ EEG, which was recorded continuously at $250 \mathrm{~Hz}$ and filtered (1-50 $\mathrm{Hz}$ ). The high- and low-frequency filters were .01 and $100 \mathrm{~Hz}$ and the signals were amplified by a factor of 50,000. EEG data were visually inspected for eye blinks and artifact and these were removed from the data collection. The data was filtered at $30 \mathrm{~Hz}$ with a $6 \mathrm{db}$ roll off for each $1 \mathrm{~s}$ epoch preceding the manual markers (approximately 10/condition). After rejecting all artifacts manually, a fast fourier transform (FFT) was used to obtain the power spectrum data (in $\mu \mathrm{V}^{2}$ ). All data was submitted to FFT transformations within the Neuroscan software. Power spectrum analysis was conducted and we used the alpha $(8-12 \mathrm{~Hz})$ frequency because the alpha frequency reflects somatosensory cortical functions. ${ }^{15}$

\section{Data Analysis}

EEG lateralization was determined by computing a coefficient using the formula: [(right - left / right + left) x 100], applied on spectral amplitude values from homologous right and left electrodes to examine hemisphere differences. A positive number represents

right hemisphere specialization and a negative number represents left hemisphere specialization.

\section{RESULTS}

As shown in Figure 1, it is highly possibility that participants exhibit right hemisphere specialization for verbal and rhythm instructions and left hemisphere specialization for melody instruction. 


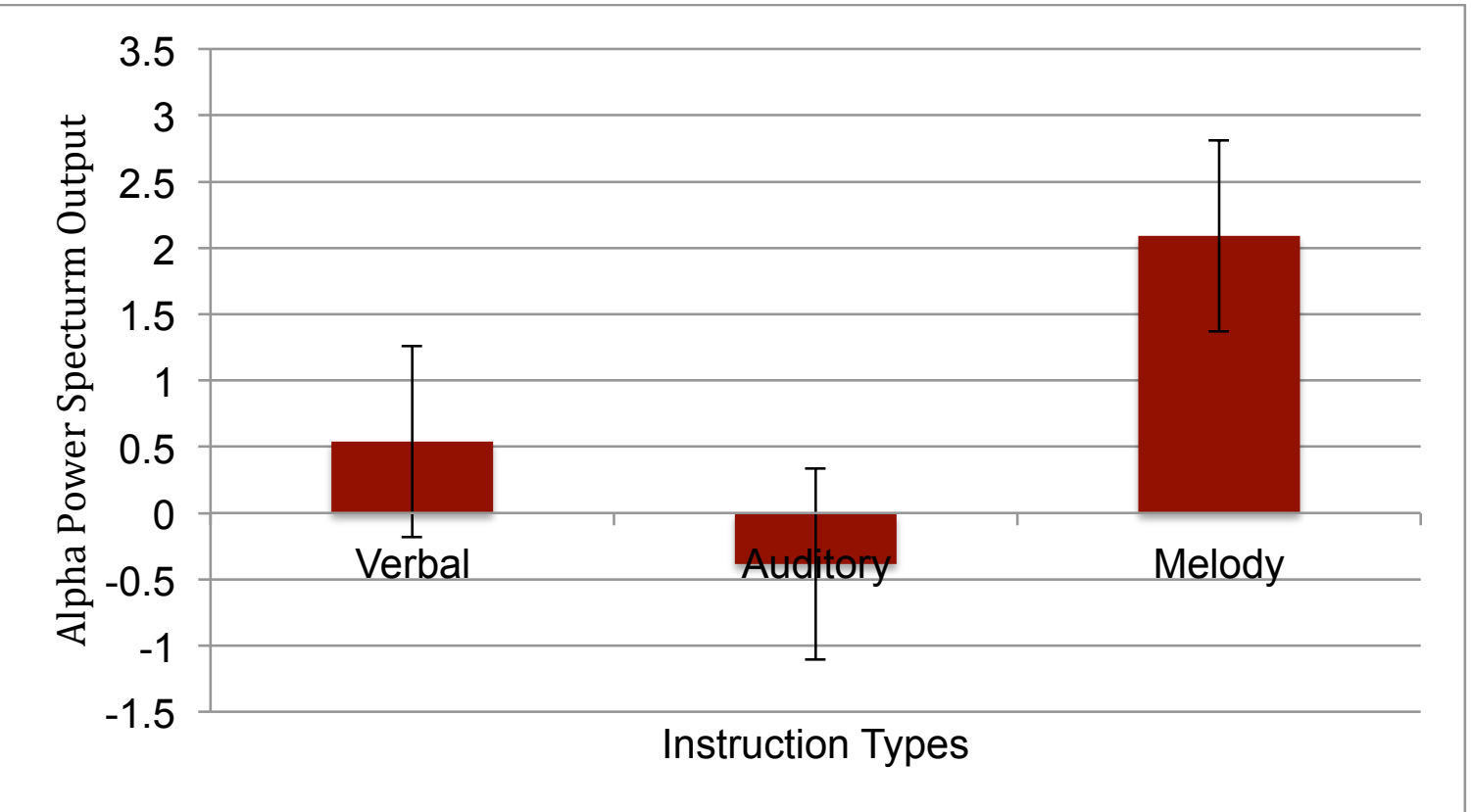

Figure 1. EEG lateralization coefficients in verbal, rhythm and melody computed using the following formula applied to absolute power amplitude: ([(right - left) / (right + left)] x 100)

\section{DISCUSSION}

This is among the first neuroimaging studies to examine how people with DS process verbal, rhythm and melody information during continuous movement performance. Our results are consistent to the previous model ${ }^{7}$ and our hypotheses that people with DS appear to exhibit a left hemisphere advantage for rhythm instruction and right hemisphere advantage for verbal. Some theories have been proposed to explain their atypical hemisphere lateralization in verbal instruction. First, Geschwind and Galaburda (1987) proposed a genetic theory and indicated the hemisphere lateralization was directly linked to delayed growth of the left hemisphere and increased growth of the right hemisphere particularly in the posterior regions in persons with DS. ${ }^{16}$ Thus, the decreased growth of the left hemisphere may be linked to the poor verbal ability in persons with DS. In addition, Previc (1991) also proposed that the atypical hemisphere model in people with DS was because of the asymmetric development of their vestibular system. ${ }^{17}$ Furthermore, this non-significant finding during verbal-motor integration may also reflect the assumption that persons with DS may employ an adapting strategy, adopted by central nervous system, to reduce the need for between-hemisphere communication. ${ }^{18}$ However, more work is still needed to understand the cerebral specialization for perceptual-motor integration in this population.

In addition, little information is known regarding music processing in persons with DS, but it was proposed that in the typical population music was processed bilaterally, with rhythm components being processed in the right hemisphere and melody components of music being processed in the left hemisphere. ${ }^{12,}{ }^{13}$ It indicates that our results are consistent with processing of music in typical persons, in which melody was primarily processed in the right hemisphere while rhythm was processed in the left hemisphere. Further, Stratford and Ching (1983) ever attempted to examine the rhythm and time in the 
perception of children with DS and their typical peers and suggested that there was no difference in rhythmic and timing discrimination between these two groups. ${ }^{19}$ Moreover, persons with DS enjoyed music and would like to move more when they heard music based on parental reports. ${ }^{20}$ Hence, it is possible that people with DS may gain benefits in motor performance while following music which incorporated into job training and the work environment because of the similar cerebral processing of music and the increased motivation. Therefore, further research should examine in more detail if there is any potential therapeutic benefits of music instruction for improving the lives of people with DS.

There are some study limitations in the current study. First, EEG data showed the atypical cerebral specialization in response to verbal-motor integration in the current study. However, the lateralization coefficients were not large in verbal and auditory instructions. It is possible that some participants with DS may not process by circuitry specialized in verbal instruction, while the verbal "drum" was rhythmically repeated. In addition, a large variability in EEG data may result from different musical experience (e.g., music therapy, music lessons and instruments) and age-related changes in EEG alpha wave among participants. ${ }^{21}$

\section{CONCLUSION}

In summary, the findings of the current study indicate that people with DS have an atypical cerebral lateralization in verbal-motor integration and the similar specializations in auditory- and music- motor integration during drumming movement. To my knowledge, this is the first study to apply EEG technique instead of behavior outcome to understand these phenomena in this population. Sensory perception capabilities, previous music experience and age should be considered in the future studies in persons with DS.

\section{REFERENCES}

1. Phelps RA, Pinter JD, Lollar DJ, Medlen JG, Bethell CD. Health care needs of children with Down syndrome and impact of health system performance on children and their families. J Dev Behav Pediatr 2012; 33: 214-20.

2. Elliott D, Edwards JM, Weeks DJ, Lindley S. Cerebral specialization in young adults with Down syndrome. American Journal of Mental Deficiency 1987.

3. Hartley XY. Receptive language processing of Down's syndrome children. Journal of Mental Deficiency Research 1982.

4. Pipe M-E. Atypical laterality and retardation. Psychological Bulletin 1988; 104: 343-9.

5. Heath M, Elliott D, Weeks DJ, Chua R. A functional systems approach to movement pathology in persons with Down syndrome. In: Weeks DJ, Chua R, Elliott D, editors.

Perceptual-Motor Behavior in Down syndrome. Human Kinetics. Champaign III. 2000. 305-20.

6. Maraj BKV, Robertson SD, Welsh TN, Weeks DJ, Chua R, Heath M, et al. Verbal-Motor Behaviour in Persons with Down Syndrome. In: Cuskelly M, Jobling A, Buckley S, editors. Down syndrome across the life span. Whurr Publishers Ltd. London, ENG. 2002. 175-93. 
7. Weeks DJ, Chua R, Weinberg H, Elliott D, Cheyne D. A preliminary study using magnetoencephalography to examine brain function in Down's syndrome. Journal of Human Movement Studies 2002; 42: 1-18.

8. Groen MA, Alku P, Bishop DVM. Lateralisation of auditory processing in Down syndrome: A study of T-complex peaks Ta and Tb. Biological psychology 2008; 79: 148-57.

9. Levitin DJ, Cole K, Chiles M, Lai Z, Lincoln A, Bellugi U. Characterizing the musical phenotype in individuals with Williams syndrome. Child Neuropsychology 2004; 10: 223-47. 10. Ringenbach SD, Gibbons Z, Chen C-C, Mulvey G. Visual-part and music instructions improve timing consistency in bimanual drumming in adults with Down syndrome. Journal of sport \& exercise psychology 2010; 32: S48.

11. Bolduc C, Daoust A-M, Limoges É, Braun CMJ, Godbout R. Hemispheric lateralization of the EEG during wakefulness and REM sleep in young healthy adults. Brain and cognition 2003; 53: 193-6.

12. Bryden MP. Laterality functional asymmetry in the intact brain. In: editors. Elsevier. 2012.

13. Hopyan T, Dennis M, Weksberg R, Cytrynbaum C. Music skills and the expressive interpretation of music in children with Williams-Beuren syndrome: pitch, rhythm, melodic imagery, phrasing, and musical affect. Child Neuropsychol 2001; 7: 42-53.

14. Oldfield RC. The assessment and analysis of handedness: the Edinburgh inventory. Neuropsychologia 1971; 9: 97-113.

15. Serrien DJ, Brown $P$. The functional role of interhemispheric synchronization in the control of bimanual timing tasks. Experimental brain research 2002; 147: 268-72.

16. Geschwind N, Galaburda AM. Cerebral lateralization : biological mechanisms, associations, and pathology. In: editors. MIT Press. Cambridge, Mass.; 1987.

17. Previc FH. A general theory concerning the prenatal origins of cerebral lateralization in humans. Psychological review 1991; 98: 299.

18. Latash ML, Anson JG. What are "normal movements" in atypical populations? Behavioral and brain sciences 1996; 19: 55-68.

19. Stratford B, Ching EYY. Responses to music and movement in the development of children with Down's syndrome. Journal of Intellectual Disability Research 1989; 33: 13-24. 20. Pratt RR. Art, dance, and music therapy. Physical medicine and rehabilitation clinics of North America 2004; 15: 827-41.

21. Devinsky O, Sato S, Conwit RA, Schapiro MB. Relation of EEG alpha background to cognitive function, brain atrophy, and cerebral metabolism in Down's syndrome: age-specific changes. Archives of Neurology 1990; 47: 58-62. 
Citation: Chen C-C (JJ), Ringenbach SDR, Biwer A, Riekena. A Cerebral Lateralization of the EEG during Perceptual-Motor Integration in Young Adults with Down Syndrome: A Descriptive Study. BJMB. 2015: 9(2): 1-7.

Editor: Joao A. C. Barros, California State University Fullerton, Fullerton, CA, USA. Copyright: (C) 2015 Chen C-C (JJ), Ringenbach SDR, Biwer A, Riekena A and BJMB. This is an open-access article distributed under the terms of the Creative Commons AttributionNonCommercial-NoDerivatives 4.0 International License which permits unrestricted use, distribution, and reproduction in any medium, provided the original author and source are credited. Competing interests: The authors have declared that no competing interests exist.

Download: http://socibracom.com/bjmb/index.php/bjmb/issue/view/25 